\title{
Confiança e Satisfação na Compra de Turismo Online
}

\author{
Trust and Satisfaction in Online Tourism Consumption
}

Confianza y Satisfacción en la Compra de Turismo Online

\author{
Ana Alice Domenech Oneto ${ }^{1}$ \\ Jorge Brantes Ferreira ${ }^{2}$ \\ Cristiane Junqueira Giovannini ${ }^{3}$ \\ Jorge Ferreira da Silva ${ }^{4}$
}

\begin{abstract}
Resumo: Apesar de sua crescente importância, ainda há uma lacuna sobre as motivações e barreiras envolvidas no comércio eletrônico de turismo. O propósito central deste estudo é avaliar o papel da confiança e seus antecedentes na formação da intenção de compra de produtos e serviços turísticos na internet. As hipóteses foram testadas via equações estruturais com base em uma survey envolvendo 292 consumidores de turismo online. Os resultados indicam relações significativas entre confiança e os demais construtos abordados, particularmente segurança e satisfação, influenciando significativamente a intenção de compra de produtos e serviços de turismo na internet.
\end{abstract}

Palavras-Chave: Confiança. Turismo. Comércio Eletrônico. Intenção de Compra. Comportamento do Consumidor.

Abstract: Despite its growing importance, there is still a gap in the literature about the motivations and barriers involved in the e-commerce of tourism-related services and products. The central purpose of this study is to evaluate trust and its antecedents role in the formation of the intention of purchasing travel products and services on the Internet. Structural equations modeling performed with data from a survey of 292 previous buyers of online tourism products and services was used to test the proposed model. The results point towards significant relationships between trust and the other addressed constructs, particularly security and satisfaction, most of which were found to significantly influence the intention to purchase tourism-related services or products online.

Keywords: Trust. Tourism. e-Commerce. Purchase Intention. Consumer Behavior.

Resumen: A pesar de su creciente importancia, hay una brecha en las motivaciones y barreras que llevan a los consumidores a utilizar el comercio electrónico para el consumo turístico. El propósito central de este estudio es evaluar el papel de la confianza y sus antecedentes en la formación de la intención de compra de productos y servicios de viajes en Internet. Las hipótesis fueron probadas a través de ecuaciones estructurales sobre la base de una encuesta de 292

1 Pontifícia Universidade Católica do Rio de Janeiro Mestre em Administração de Empresas pela PUC-Rio. E-mail: anaalice.domenech@gmail.com

2 Pontifícia Universidade Católica do Rio de Janeiro E-mail: jorgebf@gmail.com

3 Pontifícia Universidade Católica do Rio de Janeiro Doutoranda do programa de pós-graduação em Administração de Empresas da IAG/PUC-Rio. Mestre em Administração de Empresas pela PUC-Rio. E-mail: mestrekis@gmail.com

4 Pontifícia Universidade Católica do Rio de Janeiro Professor Titular do programa de pós-graduação em Administração de Empresas da IAG/PUC-Rio. Pesquisador PQ-1A do CNPQ. Presidente da Associação Nacional de Pós-Graduação e Pesquisa em Administração (ANPAD). Doutor em Engenharia Industrial pela PUC-Rio. E-mail: jorge1319@gmail.com 
consumidores online de turismo. Los resultados indican una relación significativa entre la confianza y otras construcciones abordadas, influyendo significativamente la intención de comprar productos y servicios turísticos en Internet.

Palabras clave: Confianza. Turismo. Comercio Electrónico. Intención de Compra. Comportamiento del consumidor.

\section{INTRODUÇÃO}

O turismo vem ganhando importância em todo o mundo por sua grande relevância para o desenvolvimento econômico e social, gerando renda e empregos diretos e indiretos. Segundo dados do World Travel and Tourism Council (2015), o turismo emprega diretamente mais de 150 milhões de pessoas no mundo (em torno de $3,6 \%$ de todos os empregos da economia mundial), constituindo uma indústria de mais de 7,5 trilhões de dólares anuais.

Com o aumento do número de turistas e a relevância estratégica do setor, o e-commerce assume um papel cada vez mais relevante na busca de vantagem competitiva entre as empresas de turismo. Com o uso da internet, as empresas têm a possibilidade de criar um canal de contato direto com seus clientes, podendo não só fornecer informações sobre seus produtos e serviços, como também realizar vendas diretas. De acordo com Amaro e Duarte (2015), a compra online de produtos e serviços ligados ao turismo gerou uma receita de mais de 340 bilhões de dólares em 2011. Com crescimento anual de mais de $13 \%$ desde 2011, o site eMarketer (2015), em sua World Wide Digital Travel Sales Report, afirma que as vendas digitais de turismo alcançaram uma receita mundial de mais de 450 bilhões de dólares em 2014. Porém, para assegurar o sucesso do uso da internet no relacionamento com clientes da indústria de turismo, é preciso compreender as necessidades e desejos do consumidor, identificando os fatores que promovem a intenção de utilizar a web para adquirir produtos ou serviços.

Existem na literatura diversos estudos sobre variáveis que influenciam o comportamento do consumidor online, com a confiança sendo destacada como fator de grande influência sobre intenções de uso e compra online (Amaro e Duarte, 2015; Law, Leung e Buhalis, 2009). De acordo com Ponte, CarvajalTrujillo e Escobar-Rodríguez (2015) e Kim, Chung e Lee (2011), a confiança é um dos fatores mais relevantes para realização de negócios online ligados ao turismo, impactando a disposição dos consumidores por causa de seu papel na redução da incerteza associada ao consumo na internet. Porém, apesar da crescente importância do turismo e do aumento da literatura sobre compra de turismo online (Amaro e Duarte, 2015), faltam informações sobre o que motiva os viajantes a utilizarem a rede para obter informações ou contratar produtos e serviços de turismo (Amaro e Duarte, 2015).

A partir de uma revisão da literatura sobre o assunto, este estudo busca identificar as consequências comportamentais da confiança dos clientes finais de sites de produtos e serviços de turismo. Para tanto, foi realizada uma survey com 255 consumidores de serviços ou produtos de turismo online e testado, por meio de equações estruturais, um modelo que relaciona os construtos confiança e satisfação com outros construtos relevantes para o consumo na internet (design, facilidade de uso, utilidade percebida, segurança e privacidade) e avalia seus efeitos na intenção de compra de turismo online.

\section{REVISÃO DA LITERATURA}

\subsection{Turismo na Internet}


O rápido desenvolvimento da tecnologia da informação vem provocando mudanças drásticas na indústria do turismo e transformando o turismo em um dos setores-chave do comércio eletrônico (Ponte et al., 2015). No contexto do turismo, pesquisar informações turísticas na web é uma grande tendência entre os viajantes e a facilidade de exposição de alguns produtos de viagem, como aluguel de carros ou passagens aéreas, promove a venda pela internet (Buhalis \& Licata, 2002; Buhalis \& Law, 2008).

Segundo a OMT (Organização Mundial de Turismo), o setor e a Internet são parceiros ideais. A Internet permite acesso imediato a informações relevantes sobre os destinos em todo o mundo de forma rápida e fácil. Ponte et al. (2015) destacam que produtos e serviços turísticos são perfeitamente adequados para venda online uma vez que, em geral, envolvem um nível mais elevado de intangibilidade, envolvimento e maior nível de diferenciação do que outros bens de consumo tangíveis, sendo consequentemente mais facilmente vendidos por meio da web. Do ponto de vista das empresas, isso permite que fornecedores de viagens reduzam os custos de intermediação, além de poderem atingir novos clientes e mercados. Ter um site tornou-se essencial não só para o comércio de produtos e serviços como para fortalecer o relacionamento com os clientes e aumentar o poder de alcance das empresas no mercado. O E-commerce e o marketing online estão mudando não só a forma de fazer negócios no mercado de turismo, mas também a forma como consumidores percebem os produtos e serviços (Mamaghani, 2009). Com isso, as empresas tradicionais do setor de turismo tem uma importante decisão a tomar, pois necessitam escolher entre comercializar seus produtos pela internet, permanecer atuando de acordo com o modelo de negócio tradicional ou adotar uma estratégia progressiva de mix entre os dois.

\subsection{0 consumidor de turismo online}

Segundo Buhalis e Law (2008), existe um "novo" turista, cada vez mais sofisticado e menos interessado em optar por pacotes turísticos padronizados. Esta tendência leva a um favorecimento do turismo personalizado que substitui os pacotes tradicionais. Este consumidor moderno está mais conectado, mais exigente e busca uma interação direta com o fornecedor de forma a satisfazer seus desejos e expectativas mais específicas. De acordo com Buhalis (1998), Kim et al. (2011) e Amaro e Duarte (2015), os turistas se tornaram mais independentes e sofisticados e dispõem de uma ampla variedade de ferramentas para organizar suas viagens. Estas incluem sistemas de reserva e agências de viagens online (como Expedia), ferramentas de busca (como o Google), redes sociais, portais (como o site TripAdvisor), sites de comparação de preços, sites de compra coletiva, fornecedores individuais e sites intermediários.

Apesar de todas as mudanças no perfil do consumidor e no acesso a informações trazidas pelo meio web, ainda há muito o que se estudar sobre as razões pelas quais os consumidores usam o ambiente online para a compra de produtos e serviços. Kim et al. (2011) afirmam que há poucos estudos sobre os efeitos da confiança na compra online de produtos e serviços de turismo. Amaro e Duarte (2015), por sua vez, acreditam que a inclusão da confiança em estudos sobre a intenção de compra de produtos e serviços de turismo é particularmente relevante. Weber e Roehl (1999) apontam a "confiabilidade do fornecedor de internet" como uma das características mais importantes das compras de viagens online. Já Susskind et al. (2003) e Wen (2009) apontam a interface amigável como fator crítico no processo de decisão de compra de um produto ou serviço de turismo. Apesar da comodidade na busca de informações e compras, muitos consumidores ainda preferem usar opções de compra tradicionais. As razões para os consumidores não comprarem ou planejarem produtos de viagens online são: a falta de serviço pessoal, questões de seguran- 
ça e privacidade, falta de experiência e a demora (Weber \& Roehl, 1999; Wolfe, Hsu, \& Kang, 2004; Wen, 2010; Ponte et al., 2015).

Outro aspecto importante encontrado nas pesquisas é a importância da opinião de outros consumidores de turismo na hora de tomar uma decisão. Segundo Urban et al. (2009), a busca por depoimentos e opiniões de outros usuários tem levado consumidores do mundo todo a buscar informações sobre destinos e serviços turísticos em comunidades virtuais, de forma que possam avaliar melhor as empresas, os destinos e as opções, antes de tomar uma decisão.

\subsection{Confiança online}

Intrinsecamente, a confiança implica na disposição de uma parte em aceitar vulnerabilidade, mas com a expectativa de que pode contar com a outra parte (Ponte et al., 2015). Urban et al. (2009) e Beldad, de Jong e Steehouder (2010) propõem a confiança como um elemento essencial para a construção de relacionamentos fortes com os clientes. Relações de confiança não são criadas apenas entre pessoas ou entre pessoas e organizações, mas também podem ser encontradas entre as pessoas e sistemas computacionais. Desde o nascimento da internet, a confiança tem sido reconhecida como um fator fundamental para o sucesso da web (Beldad, de Jong, \& Steehouder, 2010). Por outro lado, configura também a maior barreira para a internet comercial (Urban et al., 2009), uma vez que consumidores não podem ver, tocar ou sentir o produto que pretendem adquirir. Gefen, Karahanna e Straub (2003), assim como Escobar-Rodríguez e Carvajal-Trujilo (2014), apontam que a geração de confiança é um método eficaz de redução da incerteza no $e$ commerce e desempenha um papel crítico em processos de compra onde os consumidores procuram produtos de alta qualidade e serviços.

Um dos principais motivos de preocupação dos consumidores com compras online é a falta de contato pessoal, o face-a-face com o vendedor ou provedor do serviço (Ponte et al., 2015). Essa falta de contato, seja com o vendedor, seja com a empresa ou mesmo com o produto a ser adquirido, exige do consumidor uma disposição inicial para confiar, que influencia de maneira positiva os comportamentos e atitudes.

Em essência, a confiança é desenvolvida quando os consumidores formam impressões positivas de um site e assim se predispõem a aceitar certa vulnerabilidade (Urban et al., 2009; Ponte et al., 2015). Weber e Roehl (1999) descobriram que a confiança pode ser construída entre clientes e empresas online através da experiência positiva de transações passadas. Como resultado da falta de confiança, muitos consumidores hesitam em compartilhar informações pessoais com e-fornecedores e fazer compras pela web. Sendo assim, a falta de confiança dos consumidores tem sido um dos grandes obstáculos para o comércio eletrônico (Aldridge et al., 1997; Hoffman et al., 1999; Beldad, de Jong, \& Steehouder, 2010; Wen, 2010). Bart et al. (2005) aprofundou a investigação existente e encontrou que a confiança exerce papel de variável mediadora entre diversos antecedentes, tais como características do site e dos consumidores, e a intenção comportamental de comprar online. Por sua vez, Beldad, de Jong, \& Steehouder (2010) realizaram uma revisão de literatura sobre os antecedentes da confiança online, apontando entre eles a facilidade de uso, características gráficas de websites e garantias de segurança e privacidade.

Muitas pesquisas sugerem que a confiança afeta a intenção comportamental de compra (Shankar, Urban, \& Sultan, 2002). A confiança deve ser um mecanismo empregado para reduzir a incerteza do consumidor e assim influenciar a sua decisão. Em resumo, espera-se que a confiança, num contexto online, afete a vontade do consumidor em adquirir produtos ou serviços neste canal (Wen, 2010; EscobarRodríguez \& Carvajal-Trujillo, 2014). Particularmente, no caso da compra online de produtos e serviços de 
turismo, alguns estudos encontram impactos significativos da confiança sobre a intenção de compra (Wen, 2009; Ponte et al., 2015), enquanto outros encontram uma relação fraca entre os dois construtos (Amaro \& Duarte, 2015).

Baseando-se nisso, e no potencial de uso do ambiente online para o comércio de produtos e serviços de turismo, enuncia-se a primeira hipótese deste estudo:

Hipótese 1: A confiança possui um efeito direto e positivo sobre a intenção de compra online de produtos e serviços de turismo.

\subsection{Antecedentes da Confiança}

Uma série de fatores influencia a formação das crenças de confiança do consumidor a respeito de fornecedores na internet, afetando direta ou indiretamente suas atitudes e intenções. Compreender a natureza e os antecedentes da confiança do consumidor online pode proporcionar um melhor entendimento de suas razões para consumir produtos e serviços ligados ao turismo na internet. Confiança está ligada à percepção de segurança que os clientes têm em relação às transações que realizam. Essa segurança diz respeito à proteção dos dados do consumidor e à garantia de entrega do produto (Ponte et al., 2015). Outros estudos falam da satisfação como gerador de confiança. Nesse contexto, a confiança dependente parcialmente do grau de satisfação do cliente (Selnes, 1998; Garbarino \& Johnson, 1999; Flavián et al., 2006; Wen, 2009). Diversos autores apontam o design e o layout do site como elementos influenciadores da satisfação (Wen, 2010; Kim et al., 2011). O design seria responsável pela primeira impressão do consumidor, por isso a interface deve ser fácil de navegar e permitir acesso rápido (Shankar et al., 2002; Kim et al., 2011). Por fim, outro elemento importante é a utilidade percebida pelo cliente. Segundo Flávian et al. (2006), quanto maior a usabilidade, maior será a satisfação do cliente.

\subsection{Design do Site}

O design de sites é um conceito amplo que vem sendo estudado tanto pela literatura de sistemas de informação quanto pela literatura de marketing. Vários estudos mostram uma relação positiva entre a comunicação visual do site e a satisfação geral do usuário (Lindgaard \& Dudek, 2003; Lee \& Lin, 2005; Im \& $\mathrm{Ha}, 2011 ; 2012)$.

Wang e Emurian (2005) sugerem que características indutoras de confiança podem ser encontradas no design do site. Ou seja, características do design do site como sua dimensão gráfica, sua estrutura de organização e acessibilidade, seu conteúdo e a presença de recursos sociais, podem intensificar percepções de confiança. De particular interesse para este estudo são as dimensões do design gráfico - aparência, cor, imagens e tipologia - e do design estrutural - facilidade de navegação, facilidade de uso, simplicidade e consistência. Por sua vez, Karimov, Brengman e Hove (2011) agrupam as características gráficas e estruturais do site numa única dimensão: design visual.

A aparência de um site serve como base para os consumidores formarem uma primeira impressão do comerciante, elaborar um parecer sobre sua confiabilidade e finalmente decidirem ou não comprar ali.

Segundo Araujo, Silva e Christo (2012), o design é o elemento que agrupa todas as fases do processo de compra em um conjunto coerente, tornando-o, efetivamente, o elemento mais importante para que o usuário se localize no website e decida seus próximos passos. Urban et al (2009) afirmam que ao construir um site confiável, o design é importante porque faz com que os usuários pensem que estão em um ambi- 
ente profissional e porque a navegação em um site bonito e fácil de usar incentiva os usuários a passarem mais tempo navegando. Diversos estudos apontam o papel de navegação como um fator-chave para a geração de uma percepção de segurança durante compras online (Pearson et al., 2007; Pratt et al., 2004). Navegação e apresentação estão também diretamente relacionadas com a conveniência e facilidade de uso, fatores que geram mais confiabilidade (Belanger et al., 2002).

Wen (2009) afirma que a qualidade de um sistema, medida em termos de usabilidade, clareza, segurança, facilidade de uso e tempo de resposta, é valorizada por usuários de e-commerce no geral, e será constantemente requerida conforme mais viajantes fizerem uso da internet para adquirirem produtos e serviços de turismo. Seguindo um raciocínio similar, Kim, Chung e Lee (2011) apontam para o conceito de funcionalidade navegacional, que trata da avaliação do desempenho do website, englobando conceitos de expertise técnica, utilidade, eficácia e velocidade de resposta como possuindo um efeito positivo significante sobre percepções de segurança, confiança e satisfação. A funcionalidade navegacional associa as funções visuais e estruturais do website, tais como barras de navegação, hyperlinks e menus à utilidade do mesmo.

Elementos como cores, tipologia e layout podem contribuir para uma percepção de segurança e privacidade transmitindo uma imagem de um ambiente profissional, sério e consequentemente, confiável (Karvonen \& Parkkinen, 2001; Basso, Goldberg, Greenspan, \& Weimer, 2001; Oh, Fiorito, Cho, \& Hofacker, 2008; Ponte, Carvajal-Trujillo, \& Escobar-Rodriguez, 2015). Já o site amigável, em que o design ajuda a facilitar o uso e dispõe as informações de forma acessível, pode gerar uma percepção de um site útil e fácil de usar (Chau, Hu, Lee, \& Au, 2007; Oh, Fiorito, Cho, Hofacker, 2008; Kim \& Niehm, 2009; Luo, Ba, \& Zhang, 2012).

Neste estudo, é proposto um modelo em que a influência do design, ou seja, das características gráficas e estruturais, do site sobre a confiança e satisfação se dá indiretamente por meio da influência positiva do design sobre antecedentes da satisfação e confiança, como facilidade de uso percebida, utilidade percebida, privacidade e segurança.

Com base nessas considerações, são propostas as seguintes hipóteses:

Hipótese 2: $O$ design do site possui um efeito direto e positivo sobre a percepção de segurança dos usuários de sites de produtos e serviços de turismo.

Hipótese 3: $O$ design do site possui um efeito direto e positivo sobre a percepção de privacidade dos usuários de sites de produtos e serviços de turismo.

Hipótese 4: $\mathrm{O}$ design do site possui um efeito direto e positivo sobre a facilidade de uso percebida em sites de produtos e serviços de turismo.

Hipótese 5: $O$ design do site possui um efeito direto e positivo sobre a utilidade percebida em sites de produtos e serviços de turismo.

\subsection{Utilidade Percebida}

O construto utilidade percebida retrata o quanto um indivíduo acredita que uma dada tecnologia pode melhorar sua produtividade ou desempenho em alguma tarefa (Davis et al., 1989). Aplicando essa definição ao contexto da pesquisa, utilidade percebida refere-se à percepção dos consumidores de que o uso da internet como um meio de compras melhora o resultado da sua experiência de compra. Tal percepção influenciaria a intenção de consumidores em realizar compras na internet (Escobar-Rodríguez \& Carvajal-Trujillo, 2014). 
A utilidade percebida trata de como o site fornece informações suficientes sobre os produtos e serviços que estão sendo promovidos (Wen, 2009; Kim et al., 2011). Reflete também o quão fácil é para usuários encontrar informações relevantes e a eficiência com a qual conseguem fechar transações.

Com base nesses estudos, espera-se que a utilidade percebida tenha efeitos positivos diretos sobre a satisfação e a confiança do consumidor em relação à compra de produtos e serviços de turismo online:

Hipótese 6: A utilidade percebida possui um efeito direto e positivo sobre a confiança em sites de produtos e serviços de turismo.

Hipótese 7: A utilidade percebida possui um efeito direto e positivo sobre a satisfação relacionada a sites de produtos e serviços de turismo.

\subsection{Facilidade de Uso Percebida}

Facilidade de uso é definida como a percepção do indivíduo de que o uso da nova tecnologia será livre de esforço (Davis et al., 1989). Aplicando isso ao contexto desta pesquisa, "facilidade de uso" é a percepção do consumidor de que compras de produtos ou serviços de turismo na Internet vão envolver um esforço mínimo. A facilidade de utilização reduz o tempo de pesquisa online e de compra maximizando os esforços dos clientes e aumentando sua satisfação e sua percepção de utilidade (Wen, 2009). Segundo Xue e Harker (2002), a facilidade de uso é decisiva para a satisfação do cliente, uma vez que potencializa a eficiência de utilização do serviço. Num contexto eletrônico, a facilidade de utilização inclui aspectos como a funcionalidade, acessibilidade à informação, facilidade de encomenda e navegação. Com base nessa discussão, é possível declarar as seguintes hipóteses:

Hipótese 8: Facilidade de uso possui um efeito direto e positivo sobre a satisfação dos usuários de sites de compra e venda de produtos e serviços de turismo.

Hipótese 9: Facilidade de uso possui um efeito direto e positivo sobre a utilidade percebida do usuário de sites de compra e venda de produtos e serviços de turismo.

A literatura indica que a facilidade de utilização percebida influencia as atitudes de consumidores no que concerne a compras online (Heijden et al., 2001). Roy et al. (2001) analisou o papel da facilidade de uso afetando a confiança dos usuários de websites e alegou que a facilidade de navegação é um fator importante para avaliar a confiabilidade do site, enquanto Amaro e Duarte (2015) destacam que complexidade de um website ou sistema de compras online é um fator importante na formação da atitude de consumidores ao comprar produtos e serviços de turismo online. Muitos estudos anteriores encontraram os mesmos efeitos da facilidade de uso ou construtos similares sobre a intenção e o comportamento dos consumidores (Kim et al., 2011). Desta forma, é possível propor a seguinte hipótese:

Hipótese 10: Facilidade de uso percebida possui um efeito direto e positivo sobre a confiança dos usuários de sites de produtos e serviços de turismo.

\subsection{Segurança}

Urban, Sultan e Qalls (2000) indicam que o usuário da Internet está se tornando mais sofisticado e exigente com relação aos serviços online, com o aspecto segurança sendo um dos mais críticos na decisão final de realizar ou não uma transação. Os websites têm uma desvantagem em relação aos agentes de viagem, pois muitos consumidores ainda não se sentem confortáveis em disponibilizar informações pessoais e 
fazer compras online por não confiarem na segurança dos websites (Kim et al., 2011). De acordo com Ponte et al. (2015), segurança, juntamente com a privacidade, é a base a partir da qual se avalia a confiabilidade de um comerciante online. Ela tem a ver com a preocupação de que uma terceira parte poderia obter informação crítica sobre o indivíduo como, por exemplo, acesso ao número do cartão de crédito ou outras informações confidenciais transmitidas durante a compra de produtos e serviços via Internet. Os consumidores estão preocupados também com a legitimidade das empresas que vendem pela rede. Diversos autores, entre eles Hoffman, Novak e Peralta (1999), Aiken e Bousch (2006), Beldad, de Jong e Steehouder (2010) e Ponte et al. (2015), apontam a segurança como um dos principais motores de confiança online. Eles argumentam que o controle ambiental ou a capacidade do consumidor de controlar as ações de um fornecedor da web afeta diretamente a percepção do consumidor de segurança online.

É importante, no entanto, ressaltar que essa relação varia para as diferentes categorias de site. Quando os consumidores compram em sites de produtos ou serviços de alto envolvimento, como é o caso dos sites de viagem, normalmente os consumidores estão mais preocupados com a exposição de informações financeiras. Para esses sites, prevê-se que o impacto da segurança na confiança online é ainda maior do que para outros sites. Assim, espera-se que a segurança tenha um efeito direto sobre a confiança e um efeito também sobre a satisfação do consumidor online de produtos e serviços de turismo. Dessa forma, é possível então enunciar as seguintes hipóteses:

Hipótese 11: A segurança percebida pelo cliente possui um efeito direto e positivo sobre a satisfação dos usuários de sites de produtos e serviços de turismo.

Hipótese 12: A segurança percebida pelo cliente possui um efeito direto e positivo sobre a sensação de confiança dos usuários de sites de produtos e serviços de turismo.

\subsection{Privacidade}

Privacidade se refere à proteção contra aquisição, disseminação e divulgação de informações pessoais dos clientes e relaciona-se com a possibilidade da empresa utilizar mal a informação que lhe é disponibilizada. Segundo Ponte et al. (2015), a percepção de risco com a ausência de privacidade na Internet influencia negativamente a decisão de compra dos internautas. Eles temem que informações pessoais recoIhidas por sites sejam mal utilizadas (Hoffman et al., 1999). Além disso, a privacidade está positivamente relacionada com a avaliação dos clientes de sites (Parasuraman et al., 2005).

Além dos processos de compra, a garantia de privacidade é também fator crítico para ajudar a desenvolver no consumidor certo nível de confiança nos sistemas de comércio eletrônico. Bart et al. (2005) apontam a privacidade como um antecedente da confiança para produtos em que tanto o risco de informações quanto o envolvimento são elevados (por exemplo, sites de viagens, onde muitas informações pessoais são exigidas). Ponte et al. (2015) destacam a importância dos efeitos de privacidade e segurança sobre confiança especificamente em situações de compra de turismo online. Dessa maneira espera-se que a importância da privacidade em determinar a confiança de um site seja maior para as categorias de sites onde informações pessoais estariam em risco do que é para outras categorias de sites. Sendo assim, com base na teoria apresentada, é possível propor as seguintes hipóteses:

Hipótese 13: A privacidade possui um efeito direto e positivo sobre a percepção de segurança dos consumidores de produtos e serviços de turismo.

Hipótese 14: A privacidade possui um efeito direto e positivo sobre a confiança do consumidor de produtos e serviços de turismo. 


\subsection{Satisfação}

A satisfação é um dos conceitos mais abordados na literatura de marketing (Anderson, 1973; Oliver \& Swan, 1989). Segundo Oliver (1997), a formação da satisfação envolve a comparação entre o resultado do consumo e a expectativa do indivíduo anterior ao consumo. A satisfação desempenha um papel importante no e-commerce porque ajuda a construir a confiança do cliente em relação à empresa que oferece produtos ou serviços e afeta o desejo do consumidor de continuar a relacionar-se com aquela empresa. (Flavián et al., 2006; Garbarino \& Johnson, 1999). A satisfação está, assim, relacionada com a confiança e é considerada um antecedente desta (Garbarino \& Johnson, 1999; Selnes, 1998). Pesquisas mostram que a satisfação do cliente leva a intenções de compra e de retorno (Anderson \& Fornell, 1994; Ranaweera et al., 2008), ou seja, que a satisfação do cliente tem um impacto positivo na sua intenção de compra.

Assim, também na internet, admite-se que um dos antecedentes da confiança é a satisfação (Kim et al., 2011), com um aumento da satisfação tendo impacto positivo na confiança (Wen, 2009). Isto permite a elaboração das seguintes hipóteses:

Hipótese 15: A satisfação possui um efeito direto e positivo sobre a confiança dos usuários de sites de produtos e serviços de turismo.

Hipótese 16: A satisfação possui um efeito direto e positivo sobre as intenções de compra dos usuários de sites de produtos e serviços de turismo.

\subsection{Intenção de Compra}

Considerando uma perspectiva abrangente, a intenção de compra online tem sido considerada como o intuito comportamental de realizar transações online (Ponte et al., 2015; Amaro \& Duarte, 2015), resultado da avaliação de determinados critérios por parte dos consumidores, sendo um preditor importante do comportamento de compra.

O processo de tomada de decisão sobre as compras online se relaciona com as experiências e percepções do consumidor durante as compras de turismo. Neste contexto, a confiança do site tem uma relação significativa e positiva com intenção de compra de turismo online (Ponte et al., 2015; Amaro \& Duarte, 2015).

A partir da revisão teórica realizada, a figura a seguir apresenta o modelo proposto no presente estudo, ilustrando as relações hipotéticas apresentadas anteriormente. 


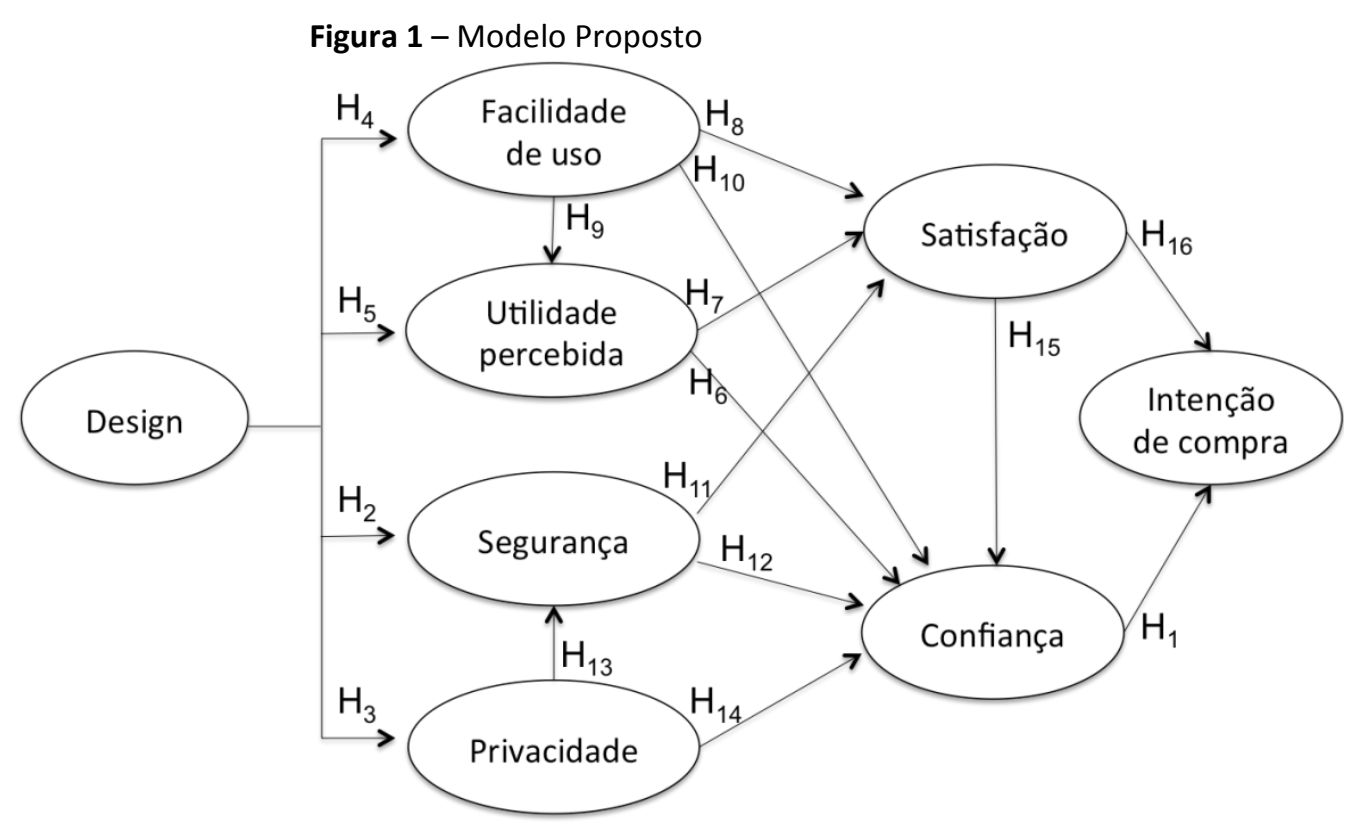

\section{METODOLOGIA}

Com o objetivo de realizar o teste das hipóteses formuladas para o estudo foi realizada uma crosssectional survey (Parasuraman et al., 2006) com uma amostra não probabilística da população de interesse, com questionários estruturados sendo apresentados a consumidores em um único momento no tempo.

A opção da coleta de dados pelo meio online teve como objetivo facilitar o acesso a um maior número de pessoas e garantir que os respondentes fossem todos usuários de Internet, uma vez que o estudo aborda a percepção de usuários a respeito de sites e serviços de turismo no ambiente virtual. Segundo a literatura, a utilização do meio virtual facilita e permite uma maior rapidez na resposta (Schaefer \& Dillman, 1998).

\subsection{Operacionalização das variáveis}

O presente estudo faz uso de escalas já elaboradas e testadas na literatura para a medição de todos os construtos envolvidos na estrutura do modelo proposto. Foi utilizada uma adaptação da E-S-QUAL de Parasuraman, Zeithaml e Malhotra (2005), com os itens sendo medidos via escalas do tipo Likert de 5 pontos, variando de "discordo totalmente" a "concordo totalmente". O instrumento de pesquisa foi composto por 52 itens, sem contar os seis itens relativos às variáveis demográficas do consumidor. Os itens incluídos no instrumento de pesquisa foram traduzidos para o português por profissionais, com etapas de tradução e retradução sendo empregadas para garantir que as escalas em português se aproximassem o máximo possível das originais. Foram realizados dois pré-testes do questionário, com o objetivo de refinar os itens e o layout do site utilizado para a pesquisa.

\subsection{Amostra e Procedimentos de Coleta de Dados}

A população estudada foi definida como usuários de sites de turismo, moradores do Rio de Janeiro, pertencentes à classe socioeconômica $\mathrm{A}$ e $\mathrm{B}$ e usaram a internet para adquirir produtos ou serviços de turismo no canal online nos últimos doze meses. Tais consumidores foram identificados por meio de uma 
pergunta filtro no instrumento de coleta. $\mathrm{O}$ objetivo desta filtragem foi obter percepções reais de usuários que já utilizaram sites de turismo, sendo assim capazes de responder com mais precisão as perguntas realizadas.

Todos os questionários foram preenchidos pelos próprios respondentes em um site online. Os dados foram coletados por meio de uma amostragem não probabilística por conveniência tipo "bola de neve". Neste método, o pesquisado indica outras pessoas de perfil similar a ele próprio para que também participem da amostra (Morgan, 2008). Para isso, um número inicial de pessoas é selecionado e é solicitado a essas pessoas informações acerca de outros membros da população de interesse para que estes sejam convidados a participar também. No caso da pesquisa em questão, foram selecionadas inicialmente cerca de 80 pessoas por meio de redes sociais (todos adultos das classes A e B que haviam realizado compras online de produtos ou serviços ligados ao turismo nos últimos 12 meses) e enviados convites via e-mail com um link para a página de pesquisa. No site da pesquisa era solicitado que o respondente encaminhasse o convite para mais 10 pessoas do seu círculo social. A pesquisa permaneceu online por seis semanas (durante os meses de janeiro e fevereiro de 2013) e foram enviados ao longo deste tempo dois lembretes via email.

Foi obtida uma amostra final de 292 respondentes. Dentre estes, 24 respondentes foram eliminados por apresentarem dados ausentes e 13 por nunca terem utilizado nenhum site de produtos e serviços de turismo. Desta forma, a amostra final foi composta por 255 questionários válidos. Deste total, 132 eram do sexo feminino (52\%) e 123 do sexo masculino (48\%). Em relação ao estado civil, 128 (50\%) respondentes se declararam solteiros e 107 (42\%) casados. No que diz respeito à renda familiar média, 114 respondentes (45\%) indicaram renda familiar mensal entre seis e dez mil reais, outros 89 (35\%) declaram renda familiar superior dez mil reais, 40 respondentes (16\%) na faixa de 3.501 a 5 mil reais e somente $5 \%$ abaixo de 3.500 reais. Já no item escolaridade, $61 \%$ possuem graduação e $38 \%$ pós-graduação. Por fim, em termos de idade, metade dos participantes (128) ficou na faixa entre 31 e 40 anos seguido de 58 respondentes (23\%) na faixa de 41 a 50 anos, $16 \%$ na faixa entre 25 e 30 anos e $11 \%$ abaixo de 25 anos ou acima de 51 anos.

\section{RESULTADOS}

\subsection{Modelo de Mensuração}

Uma análise fatorial confirmatória (CFA) com o uso do software AMOS 20.0 foi realizada para testar a validade, unidimensionalidade e confiabilidade das escalas utilizadas no modelo de mensuração.

Para avaliar o ajuste do modelo de mensuração proposto, diversos índices de ajuste foram utilizados (tanto incrementais quanto absolutos), uma vez que não existe consenso na literatura sobre qual índice (ou conjunto de índices) deve ser utilizado para checar o ajuste de modelos desta natureza (Hu \& Bentler, 1999; Schreiber et al., 2006). Após várias iterações refinando o modelo, o modelo de mensuração final, com 30 indicadores, apresentou índices de ajuste satisfatórios (RMSEA $=0,087$ com C.I. de 0,082 até 0,092; $\mathrm{CFI}=$ 0.90; IFI = 0.90; TLI =0.87; $\chi 2=1795,73$, d.f. $=620, p<0,001, \chi 2 /$ d.f. $=2,89$ ).

A validade de face para todas as escalas utilizadas foi garantida durante o desenvolvimento do instrumento de pesquisa (escolha de escalas já utilizadas na literatura, tradução cuidadosa e pré-testes). Para verificar a validade nomológica foi analisada a matriz de correlação entre construtos, com todas as correlações sendo significativas e estando na direção esperada. No que diz respeito à validade convergente, foi calculada a variância extraída média para cada construto (AVE). Todos os valores de AVE calculados esta- 
vam entre 0,62 e 0,86, evidenciando a validade convergente das escalas utilizadas. Com relação à consistência interna e confiabilidade das escalas utilizadas, todas as escalas utilizadas atenderam aos níveis mínimos de confiabilidade considerados adequados pela literatura (Fornell \& Larcker, 1981), com todas apresentando valores entre 0,87 e 0,95 para o coeficiente alfa e entre 0,87 e 0,95 para a confiabilidade composta. Por fim, todas as variâncias compartilhadas foram inferiores à variância extraída pelos itens que medem os construtos, indicando validade discriminante adequada.

\subsection{Modelo Estrutural}

A modelagem de equações estruturais (SEM) foi utilizada por meio do software AMOS 20.0 para testar o modelo proposto e as hipóteses da pesquisa. Todos os índices indicaram um bom ajuste do modelo aos dados. A razão $\chi 2 /$ d.f. foi de 3,3, ligeiramente acima do valor de 3,0 sugerido por Byrne (2010). Além disso, os índices de ajuste incrementais também se mostraram satisfatórios, com CFI de 0,90, TLI de 0,89 e IFI de 0,90. Por sua vez, os índices de ajuste absoluto apresentaram valores próximos ao limite de 0,08 estabelecido pela literatura (Hu \& Bentler, 1999; Byrne, 2010; Hair et al., 2009), indicando também um ajuste razoável do modelo. O RMSEA foi de 0,09 (C. I. de 0,085 a 0,095) e o SRMR foi de 0,010. Dados os índices apresentados, conclui-se que o ajuste do modelo proposto é satisfatório.

Após a verificação do ajuste dos modelos de mensuração e estrutural propostos, foram avaliados os coeficientes estimados para as relações causais entre os construtos (Tabela 1). A verificação de cada uma das hipóteses da pesquisa foi realizada com a análise da magnitude, direção e significância dos coeficientes padronizados estimados por meio do modelo estrutural (Byrne, 2010).

Tabela 1 - Coeficientes Padronizados Estimados, Hipóteses e Significâncias

\begin{tabular}{lccc}
\hline Relação Proposta & $\begin{array}{c}\text { Coeficiente } \\
\text { Padronizado }\end{array}$ & $\boldsymbol{p}$-value & $\begin{array}{c}\text { Hipótese } \\
\text { Verificada }\end{array}$ \\
\hline H1: Confiança $\rightarrow$ Intenção de Compra & 0,35 & $<0,001$ & SIM \\
H2: Design $\rightarrow$ Segurança & 0,06 & 0,256 & NÃO \\
H3: Design $\rightarrow$ Privacidade & 0,48 & $<0,001$ & SIM \\
H4: Design $\rightarrow$ Facilidade de Uso & 0,62 & $<0,001$ & SIM \\
H5: Design $\rightarrow$ Utilidade & 0,13 & 0,057 & NÃO \\
H6: Utilidade $\rightarrow$ Confiança & $-0,06$ & 0,427 & NÃO \\
H7: Utilidade $\rightarrow$ Satisfação & 0,49 & $<0,001$ & SIM \\
H8: Facilidade de Uso $\rightarrow$ Satisfação & 0,37 & $<0,001$ & SIM \\
H9: Facilidade de Uso $\rightarrow$ Utilidade & 0,62 & $<0,001$ & SIM \\
H10: Facilidade de Uso $\rightarrow$ Confiança & $-0,04$ & 0,484 & NÃO \\
H11: Segurança $\rightarrow$ Satisfação & 0,22 & $<0,001$ & SIM \\
H12: Segurança $\rightarrow$ Confiança & 0,51 & $<0,001$ & SIM \\
H13: Privacidade $\rightarrow$ Segurança & 0,69 & $<0,001$ & SIM \\
H14: Privacidade $\rightarrow$ Confiança & $-0,03$ & 0,554 & NÃO \\
H15: Satisfação $\rightarrow$ Confiança & 0,68 & $<0,001$ & SIM \\
H16: Satisfação $\rightarrow$ Intenção de Compra & 0,43 & $<0,001$ & SIM \\
\hline
\end{tabular}

\section{DISCUSSÃO}

Primeiramente, destaca-se o fato de que todas as hipóteses verificadas apresentaram valor $p<$ 0,001 , indicando efeitos estatísticos fortemente significativos. As magnitudes apresentadas pelas hipóteses confirmadas pela análise também se mostraram significativas em termos práticos, com valores acima dos 
0,30 . As magnitudes encontradas para os efeitos da satisfação sobre a confiança $(0,68)$, do design sobre a facilidade de uso $(0,62)$ e da facilidade sobre a utilidade $(0,62)$ se mostraram especialmente altas, apontando para uma relação forte e prática entre os construtos. As duas variáveis que apresentaram relações mais fortes em relação à confiança foram segurança e satisfação (com efeitos de magnitude de 0,51 e 0,68, respectivamente), o que está de acordo com outros trabalhos encontrados na literatura (Kim et al., 2011). A confiança está relacionada positivamente à satisfação, e ambas exercem impactos positivos de razoável magnitude sobre a intenção de compra (magnitude de 0,43 para o efeito da satisfação sobre intenção e magnitude de 0,35 para o efeito da confiança sobre a satisfação). De fato, satisfação e confiança são antecedentes da intenção de compra (Amaro \& Duarte, 2015; Ponte et al., 2015) assim como a satisfação é antecedente direto da confiança (Kim et al., 2011), apresentando uma das relações mais significativa de todo o modelo proposto (magnitude de 0,68 ). Ainda em relação à satisfação, foram verificadas também as ligações diretas e positivas e de magnitudes moderados com os construtos facilidade de uso $(0,37)$, utilidade percebida $(0,49)$ e segurança $(0,22)$ (Kim et al., 2011).

Em relação à confiança, a privacidade e a facilidade de uso não apresentaram efeitos significativos, resultados também encontrados por Ponte et al. (2015) para privacidade. No entanto, é importante ressaltar que a facilidade de uso apresentou relação significativa sobre a utilidade percebida e que a privacidade apresentou uma forte relação com a segurança, que por sua vez, teve um impacto direto e positivo sobre a confiança.

Dadas tais análises pode-se alegar que confiança e satisfação são antecedentes à intenção de compra, ou seja, quanto mais satisfeito o consumidor está com o site, mais confiança ele tem e com isso, maior sua intenção de compra.

No modelo proposto, esperava-se a relação direta do design com quatro construtos: facilidade de uso, utilidade, privacidade e segurança. Entretanto, só foram significativas as relações com os construtos privacidade (magnitude de 0,48 ) e facilidade de uso (magnitude de 0,62 ). Em relação ao uso do site, podese dizer que o design afeta a percepção da facilidade de uso do site, ou seja, quanto mais fácil de usar o consumidor percebe o site, mais útil crê que o site seja. Os resultados também mostram que o design influencia a percepção de privacidade e esta aumenta a percepção do usuário em relação à segurança.

Outra relação direta e muito significativa $(p$-valor $<0,001)$ foi da segurança sobre a confiança (magnitude de 0,51 ), indicando que na percepção do usuário, um site seguro é confiável, com a confiança promovendo a intenção de compra do usuário.

Nota-se que, para os dados do estudo, o modelo proposto explica aproximadamente $56 \%$ da variância observada na intenção de consumidores em realizar compras de turismo online. Isto indica que os construtos utilizados e relações propostas são relevantes para o comportamento de consumo estudado e conseguem capturar boa parte da variação da intenção de compra de turismo online dos consumidores avaliados. Da mesma forma, satisfação e confiança apresentam altos níveis de proporção da variância explicada ( $77 \%$ e $85 \%$, respectivamente), fato que corrobora a posição mediadora destes construtos no modelo, com ambos mediando os efeitos das avaliações cognitivas dos consumidores a respeito de design, segurança, privacidade, utilidade e facilidade sobre a intenção de compra. Por sua vez, o design sozinho foi capaz de explicar $23 \%$ da variância da percepção de privacidade dos consumidores e $38 \%$ da variância da facilidade de uso percebida, mostrando a relevância de aspectos de interface e navegação na percepção de um e-commerce ligado ao turismo. Por fim, segurança e utilidade percebida tiveram em torno de $50 \%$ de suas variâncias explicadas, respectivamente, por privacidade e facilidade de uso, indicando a relevância 
destes antecedentes na formação das percepções sobre segurança e utilidade dos consumidores de turismo online.

\section{CONCLUSÕES E CONTRIBUIÇÕES}

Estudos que analisam e buscam compreender o comportamento do consumidor têm importância fundamental para estratégias de empresas que querem conquistar clientes. Para empresas que precisam se manter competitivas, é relevante entender o consumidor e as variáveis que influenciam direta ou indiretamente seu comportamento de compra. Especificamente, com relação ao mercado de turismo, a confiança no fornecedor online foi apontada como uma das características mais importantes das compras de viagens online por Ponte et al. (2015) e Amaro e Duarte (2015). Além disso, Urban et al. (2009) e Escobar-Rodríguez \& Carvajal-Trujillo (2014) destacam a importância da confiança como um fator-chave para o sucesso da web e como a maior barreira da internet comercial, uma vez que os consumidores não podem ver, tocar ou sentir o produto que pretendem adquirir.

Os resultados e as relações verificadas respondem às questões propostas pela pesquisa e representam contribuições importantes para o entendimento mais completo do contexto online na venda de produtos e serviços de turismo. A primeira contribuição deste estudo se refere à proposição do design do website como influenciador de alguns dos antecedentes da confiança e da satisfação. Os resultados indicam que o design, gráfico e estrutural de um website influencia as percepções de privacidade e facilidade de uso do mesmo. Por sua vez, estas têm impactos diretos sobre as percepções de segurança (que antecede tanto confiança quanto satisfação) e a satisfação. Estudos anteriores apontam para a influência do design, diretamente, sobre a confiança e a satisfação (Karimov, Brengman \& Hove, 2011; Kim et al., 2011), mas a sua influência do design sobre outros construtos formativos da confiança e satisfação foi pouco estudada, e nunca sob este enfoque específico (Karimov, Brengman \& Hove, 2011; Im \& Ha, 2012).

A segunda contribuição se refere à discussão atual sobre o impacto da confiança sobre a intenção de compra online de produtos e serviços de turismo. Os resultados apontam para um efeito positivo e significativo da confiança, diferente dos resultados encontrados por Amaro e Duarte (2015), onde os resultados se mostraram estatisticamente significantes, mas fracos em magnitude. A magnitude do efeito obtida neste estudo $(0,35)$ indica que a confiança exerce efeito suficiente sobre a intenção de compra para ser considerada um fator chave na estruturação de websites de venda de produtos e serviços de turismo.

A terceira contribuição diz respeito ao impacto inteiramente mediado por confiança e satisfação que diversos construtos de avaliação cognitiva exercem sobre compras online de produtos e serviços de turismo. Da mesma forma, foi verificado que as percepções de privacidade são antecedentes das percepções de segurança, mas não de confiança, indicando que o impacto das percepções do consumidor sobre questões de privacidade é inteiramente mediado pela percepção da segurança oferecida pelo website.

Baseando-se nos resultados da pesquisa e na literatura apresentada (Ponte et al., 2015), podemos concluir que a segurança exerce uma influência positiva na intenção do consumidor de confiar e com isso estabelecer ou não um relacionamento com o site ou a empresa. Pode-se entender dessa forma que a segurança é um elemento-chave na construção de relacionamentos entre consumidor e empresas online de turismo. Entre os dois antecedentes diretos da intenção de compra, a satisfação apresentou um efeito maior do que a confiança, porém relativamente próximos. A satisfação também se mostrou um forte influenciador da confiança do consumidor online, o que indica que o consumidor satisfeito tende a confiar.

Percebe-se também como a privacidade dos dados influencia a percepção da segurança do consu- 
midor e como um site fácil de usar influencia na percepção de quão útil ele é. Podemos concluir também que a intenção de compra está relacionada mais especificamente com experiências individuais, por sentimentos diretos, como confiança, satisfação e segurança.

Quanto às implicações gerenciais para empresas que operem websites de venda de produtos e serviços de turismo o estudo pode oferecer algumas sugestões. Primeiramente, o design do site deve apresentar alta qualidade, tanto visualmente quanto estruturalmente. Sites considerados bons podem fortalecer a confiança do consumidor e aumentar sua satisfação. Sites com designs de qualidade e eficientes são sinais de que a empresa dá importância à segurança e a funcionalidade, uma vez que a aparência serve muitas vezes como proxy para o tempo e dinheiro investidos na criação do website (Ponte et al., 2015). Além disso, bons sites atraem novos clientes e ajudam o cliente recorrente a reconhecer a empresa e fazer novas compras (Wen, 2009). Websites que apresentam conteúdo textual de qualidade com fontes legíveis e cores discretas, fotos ilustrativas e textos organizados em estruturas práticas e metódicas criam imagens de profissionalismo e confiança (Karvonen \& Parkkinen, 2001). Tais características são especialmente importantes na indústria de turismo, uma vez que destinos, hotéis e eventos devem ser descritos com riqueza de informação e detalhes, tanto via texto, quando via imagens e vídeos. Da mesma forma, a busca por opções de destinos, passagens, hotéis e preços deve ser facilitada e estimulada via o design de sites de turismo, auxiliando o consumidor a construir sua viagem personalizada de forma fácil e eficiente.

Em segundo, intenção de compra é uma função da confiança e da satisfação, que dependem da funcionalidade do website. Facilidade de uso, o encontro de serviços e produtos por meio de processos de busca simples e descomplicados, utilidade, o encontro de produtos e serviços desejados ou alinhados com o que se deseja - assim como o contato com canais de avaliação e informação detalhada sobre esses produtos - e segurança, representada por sites blindados e políticas claras de privacidade e uso de informações financeiras, são sinais evidentes da importância que a empresa dá ao consumidor e aos serviços e produtos que vende.

Por fim, websites de venda de produtos e serviços de turismo devem considerar seriamente a inclusão de links relacionais em seus designs estruturais. Sites de serviços como o Facebook, ou até mesmo blogs, oferecem canais cada vez mais significativos de relacionamento com o cliente. Incluí-los na estrutura de funcionamento do site estimularia a interação do consumidor com a empresa e o fluxo de informações pertinentes para um melhor posicionamento e segmentação do público que faz uso do website (Araujo et al., 2012). p

\section{LIMITAÇÕES E PESQUISAS FUTURAS}

Uma limitação do estudo diz respeito à coleta e ao tratamento dos dados. Em relação à validade externa dos resultados, dada a amostra de conveniência por bola de neve, é possível que as relações verificadas na pesquisa não sejam generalizáveis para todo e qualquer tipo de consumidor. No que diz respeito ao procedimento de coleta de dados, uma limitação importante do trabalho foi o fato dos respondentes avaliarem sites de sua preferência e uso pessoal, sem ter sido exigida a avaliação de um site único por todo grupo. Isso limita o estudo já que o site escolhido por cada respondente tem características próprias que não puderam ser vistas e analisadas igualmente pelos outros, dando margens a avaliações bastante distintas, principalmente no que se relaciona a atributos como facilidade de uso, utilidade percebida e design.

Com relação a pesquisas futuras, a replicação do modelo proposto junto a consumidores de um site específico, para que todos pudessem avaliar um mesmo modelo, seria uma boa forma de validar e ampliar 
o escopo dos resultados aqui obtidos. Pesquisas futuras podem verificar também a influência que a marca do site exerce sobre o usuário e sua avaliação em relação aos serviços oferecidos pelo site. Outra sugestão seria verificar em uma futura pesquisa a importância de outros antecedentes de confiança e intenção de compra no ambiente online do segmento de turismo. Pesquisas futuras podem explorar também outras escalas para os construtos utilizados no modelo ou construtos conceitualmente similares, comparando seus resultados com os obtidos aqui. Por fim, seria interessante a investigação de possíveis efeitos moderadores que algumas variáveis demográficas (como sexo, renda e idade) poderiam apresentar sobre as relações observadas.

\section{REFERÊNCIAS}

Amaro, S., \& Duarte, P. (2015). An integrative model of consumers' intentions to purchase travel online. Tourism Management, 46, 64-79.

Anderson, E. W., \& Fornell, C. (1994). A customer satisfaction research prospectus. In: Rust, R. T.; Oliver, R. L. (Eds.). Service quality: new directions in theory and practice. Thousand Oaks: Sage Publications, 241-68.

Anderson, R. (1973). Consumer Dissatisfaction: The Effect of Disconfirmed Expectancy on Perceived Product Performance. Journal of Marketing Research, 10(1), 38-44.

Bart, Y., Shankar, V., Sultan, F., \& Urban, G.L. (2005). Are the drivers and role of online trust the same for all web sites and consumers? A large-scale exploratory empirical study. Journal of Marketing, 69(October), 133-152.

Araujo, R.M., Silva, A. M., \& Christo, R. S. C. (2012). Turismo na era on-line: um estudo sobre e-marketing em agências de viagens de Natal/RN. Holos, 28(4), 199-215.

Basso, A., Goldberg, D., Greenspan, S., \& Weimer, D. (2001). First Impressions: Emotional and cognitive factors underlying judgments of trust e-commerce. In Proceedings of the $3^{\text {rd }}$ ACM Conference on Electronic Commerce, 137-143. Tampa, FL, USA.

Beldad, A., de Jong, M., Steehouder, M. (2010). How shall I trust the faceless and the intangible? A literature review on the antecedents of online trust. Computers in Human Behavior, 26, 857-869.

Buhalis, D. (1998). Strategic use of information technologies in the tourism industry. Tourism Management, 19(5), 409-421.

Buhalis, D.; \& Licata, M. C. (2002). The future eTourism intermediaries. Tourism Management, 23(3), 207220.

Buhalis, D.; \& Law, R. (2008). Progress in information technology and tourism management: 20 years on and 10 years after the Internet. Tourism Management, 29(4), 609-623.

Byrne, B. (2010). Structural Equation Modeling with AMOS: Basic Concepts, Applications and Programming. 2 ed. Routledge, NY.

Davis, F., Bagozzi, R., \& Warshaw, P. (1989). User Acceptance of Computer Technology: A Comparison of Two Theoretical Models. Management Science, 35(8), 982-1002. 
eMarketer. (2015). World Wide Digital Travel Sales: eMarketer's Estimates and Forecast. Acessado 05 de Abril de 2015, em http://www.emarketer.com/Corporate/Coverage\#/results/1274/report.

Escobar-Rodríguez, T., \& Carvajal-Trujillo, E. (2014). Online purchasing tickets for low cost carriers: an application of the unified theory of acceptance and use of technology (UTAUT) model. Tourism Management, 43(August), 70-88.

Flavián, C., Guinalíu, M., \& Gurrea, R. (2006). The role played by perceived usability, satisfaction and consumer trust on website loyalty. Information \& Management, 43(1), 1-14.

Fornell, C., \& Lacker, D. F. (1981). Evaluating Structural Equation Models with Unobservable Variables and Measurement Error. Journal of Marketing Research, 18(February), 39-50.

Garbarino, E., \& Johnson, M. (1999). The Different Roles of Satisfaction, Trust, and Commitment in Customer Relationships. Journal of Marketing, 63(2), 70- 87.

Gefen, D., Karahanna, E., \& Straub, D. W. (2003). Trust and TAM in online shopping: An integrated model, MIS Quarterly, 27(1), 51-90.

Hair, J. F., Black, W. C., Babin, B. J., \& Anderson, R. E. (2009). Multivariate Data Analysis, ed. 7. Upper Saddle River: Prentice-Hall.

Heijden, H., Verhagen, T., \& Creemers, M. (2001). Predicting online purchasing behavior: Replications and tests of competing models, Proceedings of the 34th Hawaii International Conference System Sciences, Maui, HI.

Hoffman, D. L., Novak, T. P., \& Peralta, M. (1999). Building consumer trust online, Communications of the ACM, 42(4), 80-85, 1999.

Hu, L., \& Bentler, P. M. (1999). Cutoff criteria for fit indexes in covariance structure analysis: Conventional criteria versus new alternatives. Structural Equation Modeling, 6, 1-55.

Im, H., \& Ha, S. (2011). An exploration of the cognitive-affective model of satisfaction in a shopping context: a testing of competing models. The Service Industries Journal, 31(13), 2273-2278.

Im, H., \& Ha, S. (2012). Role of web site design quality in satisfaction and word of mouth generation. Journal of Service Management, 23(1), 79-96.

Karimov, F. P., Brengman, M., \& Hove, L. V. (2011). The effect of website design dimensions on initial trust: a synthesis of the empirical literature. Journal of Electronic Commerce Research, 12(4), 272- 301.

Karvonen, K., \& Parkkinen, J. (2001). Signs of Trust. In Proceedings of HCl International 2001 (First International Conference on Universal Access in Human-Computer Interaction), 1, 1076-1080.

Kim, M., Chung, N., \& Lee, C. (2011). The effect of perceived trust on electronic commerce: Shopping online for tourism products and services in South Korea. Tourism Management, 32(April), 256-265.Kim, H., \&

Niehm, L. S. (2009). The impact of website quality on information quality, value, and loyalty intentions in apparel retailing. Journal of Interactive Marketing, 23(3), 207-284. 
Law, R., Leung, R., \& Buhalis, D. (2009). Information technology applications in hospitality and tourism: a review of publications from 2005 to 2007. Journal of Travel \& Tourism Marketing, 26(5-6), 599-623.

Lee, G. G., \& Lin, H. F. (2005). Customer Perceptions of e-service Quality in Online Shopping. International Journal of Retailing \& Distribution Management, 33(2), 161-176.

Lindgaard, G., \& Dudek, C. (2003). What is this Evasive Beast We Call User Satisfaction? Interacting with Computers, 15, 429-52.

Luo, J., Ba, S., \& Zhang, H. (2012). The Effectiveness of Online Shopping Characteristics and Well-Designed Websites on Satisfaction. MIS Quarterly, 36(4), 1131-1144.

Mamaghani, F. (2009). Impact of E-commerce on Travel and Tourism: An Historical Analysis. International Journal of Management, 26(3), 365-375.

Morgan, D. L. (2008). The SAGE Encyclopedia of Qualitative Research Methods. SAGE Publications.

Oh, J., Fiorito, S. S., Cho, H., \& Hofacker, C. F. (2008). Effects of design factors on store image and expectation of merchandise quality in web-based stores. Journal of Retailing and Consumer Services, 15, 237-249.

Oliver, R. (1997). Satisfaction: a behavioral perspective on the consumer. New York: Irwin / McGraw-Hill. Oliver, R., \& Swan, J. E. (1989). Consumer Perceptions of Interpersonal Equity and Satisfaction in Transactions: Field Survey Approach. Journal of Marketing, 53(April), 21-35.

Parasuraman, A., Zeithaml, V., \& Malhotra, A. (2005). E-S-QUAL: a multiple-item scale for assessing electronic service quality. Journal of Retailing, 64(1), 12-40.

Parasuraman, A.; Grewal, D. \& Krishnan, R. (2006). Marketing Research. 2. ed., South Western College Pub.

Pearson, J.M., Pearson, A., \& Green, D. (2007). Determining the importance of key criteria in web usability. Management Research News, 30(11), 816-28.

Ponte, E.B., Carvajal-Trujillo, E., \& Escobar-Rodríguez, T. (2015). Influence of trust and perceived value on the intention to purchase travel online: Integrating the effects of assurance on trust antecedentes. Tourism Management, 47, 286-302.

Pratt, J., Mills, R., \& Kim, Y. (2004). The effects of navigational orientation and user experience on user task efficiency and frustration levels. Computer Information Systems, 44(4), 93-100.

Ranaweera, C., Bansal, H., \& McDougall, G. (2008). Web site satisfaction and purchase intentions: impact of personality characteristics during initial web visit. Managing Service Quality, 18(4), 329-48.

Roy, M.C., Dewit, O., \& Aubert, B.A. (2001). The impact of interface usability on trust in web retailers, Internet Research: Electronic Networking Applications and Policy, 11(5), 388-98.

Schaefer, D., \& Dillman, D. (1998). Development of a Standard E-Mail Methodology, Results of an Experiment, Public Opinion Quaterly, 62(3), 378-397. 
Schreiber, J. B., Stage, F. K., King, J., Nora, A., \& Barlow, E. A. (2006). Reporting Structural Equation Modeling and Confirmatory Factor Analysis Results: A Review. Journal of Educational Research, 99(6), 323-337.

Selnes, F. (1998). Antecedents and consequences of trust and satisfaction in buyer-seller relationships. European Journal of Marketing, 32, 305-22.

Shankar, V., Urban, G.L., \& Sultan, F. (2002). Online trust: a stakeholder perspective, concepts, implications, and future directions. Journal of Strategic Information Systems, 11, 325-344.

Sperber, A. D. (2004). Translation and validation of study instrument for cross- cultural research. Gastroenterology, 126(1), 124-128.

Susskind, A., Bonn M., \& Dev, C. (2003). To Look or Book: An Examination of Consumers' Apprehensiveness Toward Internet Use, Journal of Travel Research, 41(3), 256-265.

Urban, G., Sultan, F., \& Qualls, W. (2000). Placing Trust at the Center of Your Internet Strategy. Sloan Management Review, 42(1) 39-48.

Urban, G. L., Amyx, C., \& Lorenzon, A. (2009). Online Trust: State of the Art, New Frontiers, and Research Potential. Journal of Interactive Marketing, 23(2), 179-190.

Vila, N., \& Kuster, I. (2011). Consumer feelings and behaviours towards well designed websites. Information \& Management, $48,166-177$.

Wang, Y. D., \& Emurian, H. H. (2005). An overview of online trust: Concepts, elements, and implications. Computers in Human Behavior, 21, 105-125.

Weber, K., \& Roehl, W. S. (1999). Profiling People Searching for and Purchasing Travel Products on the World Wide Web. Journal of Travel Research, 37(3), 291-298.

Wen, I. (2009). Factors affecting the online travel buying decision: a review. International Journal of Contemporary Hospitality Management, 21(6), 752-765.

Wen, I. (2010). Online travelers' decision makings: a new equation model to evaluate impacts of website, search intention, and trust. Information technology \& Tourism, 1292), 153-173.

Wolfe, K., Hsu, C., \& Kang, S. K. (2004). Buyer Characteristics among users of various travel intermediaries. Journal of Travel and Tourism Marketing, 17, 51-62.

World Travel and Tourism Council (2015). Travel \& Tourism: Economic Impact 2015 World. Acessado 05 de Abril de 2015, em http://www.wttc.org/-

Lmedia/files/reports/economic\%20impact\%20research/regional\%202015/world2015.pdf

Xue, M., \& Harker P. (2002). Customer efficiency: concept and its impact on e-business management, Journal of Service Research, 4(4), 253-67. 